\title{
Changes in ATP Concentration Triggered by Chemoreception in the Plasmodia of the Myxomycete Physarum polycephalum
}

\author{
By TATSUMI HIROSE,* TETSUO UEDA \\ AND YONOSUKE KOBATAKE \\ Faculty of Pharmaceutical Sciences, Hokkaido University, Sapporo, Japan
}

(Received 13 May 1980; revised 23 June 1980)

\begin{abstract}
The intracellular ATP concentration in plasmodia of the myxomycete Physarum polycephalum was determined by the luciferin-luciferase method after stimulation with various chemotactic chemicals. Repellent salts induced an ATP increase. The change in ATP concentration paralleled changes in contraction and in electrical potential with respect to both time course and concentration dependence. Other repellents (certain organic chemicals and high osmolarity) did not induce appreciable change in ATP concentration within $15 \mathrm{~min}$, but prolonged treatment with hydrophobic repellents and $\mathrm{D}_{2} \mathrm{O}$ led to diminished ATP. None of the attractants examined (e.g. glucose, alanine, $\mathrm{KH}_{2} \mathrm{PO}_{4}$ and 2-deoxyglucose) changed the ATP concentration. A possible role of ATP as a mediator in response to salts is discussed.
\end{abstract}

\section{INTRODUCTION}

Plasmodia of Physarum polycephalum react to various chemical stimuli and exhibit positive or negative chemotaxis (Carlile, 1970; Knowles \& Carlile, 1978; Chet et al., 1977; Kincaid \& Mansour, 1978, 1979; Ueda et al., 1975; Ueda \& Kobatake, 1977a, b; Ataka et al., 1978). External chemical stimuli are first recognized at the surface membrane. Conformational change of the receptive membrane in the recognition processes has been demonstrated by studying changes in zeta potential (Hato et al., 1976 a; Ueda \& Kobatake, 1977a), adhesiveness (Ishida et al., 1977), reactivity with chemical reagents (Ishida et al., 1978) and fluorescence signals (Ueda \& Kobatake, 1979). Positive and negative taxes are found to be correlated with relaxation and contraction in the plasmodial strand, respectively (Ueda et al., 1976; Hato et al., 1976b). However, the mechanisms or molecules which mediate the passage of the information from the surface membrane to the contractile system are little understood. Involvement of $\mathrm{Ca}^{2+}$ (Mito et al., 1980) or cyclic AMP phosphodiesterase (Kincaid \& Mansour, 1979) has been suggested, but the evidence was indirect. We have compared the intracellular ATP content in the plasmodium before and after chemotactic stimulation; these and other results suggest that the intracellular ATP works as a mediator in the transduction mechanism in the plasmodium.

\section{METHODS}

Plasmodia. Except where otherwise indicated plasmodia of an American strain of Physarum polycephalum provided by Professor N. Kamiya, National Institute for Basic Biology, Okazaki, Japan, was used. A second strain, an albino (LU647 × LU861) differing in chemotactic sensitivity, was kindly supplied by Dr M. Carlile, Imperial College, London. The plasmodia were cultured by the method of Camp (1936) and allowed to crawl overnight on wet filter paper without feeding.

Chemicals. Firefiy Lantern Extract (FLE-50) which contained luciferin and luciferase was purchased from Sigma. All chemicals used as stimuli were of analytical grade. 


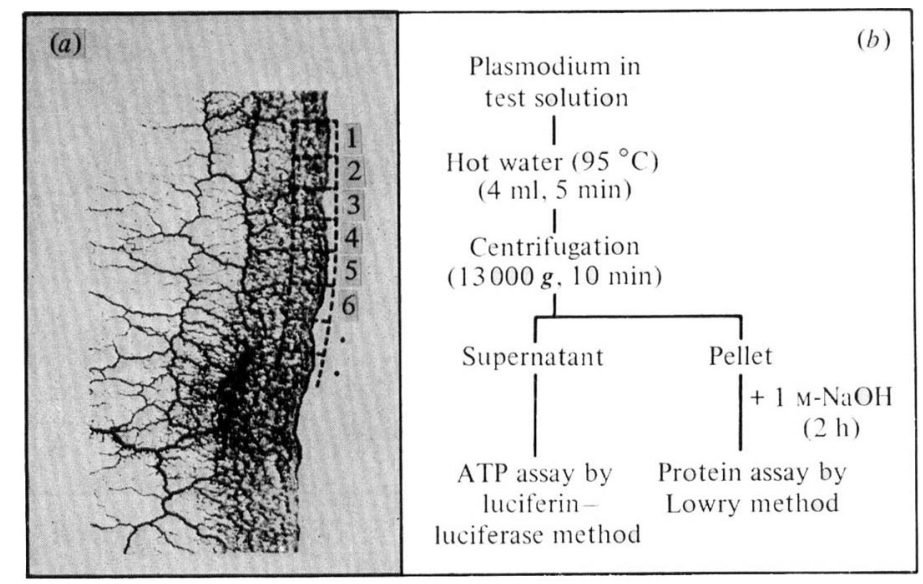

Fig. 1. (a) Advancing edge of a plasmodium; numters indicate samples removed for experiment. (b) Procedures for measuring the intracellular ATP concentration of the samples.

Measurement of intracellular ATP concentration. A single large plasmodium was collected on a filter paper. The frontal region of the advancing plasmodium was cut into several pieces $(0.5 \times 1 \mathrm{~cm})$ together with the filter paper (Fig. 1a). After standing for 15 to $30 \mathrm{~min}$ to allow recovery from possible damage, each piece was immersed in a test or control solution of $1 \mathrm{~cm}$ depth. The ATP assay procedures are indicated in Fig. 1 $(b)$ and are based on the method of Beutler \& Baluda (1964). The chemiluminescence emitted in the ATP assay was measured by a photometer (American Instrument Co., J4-7441). The maximum chemiluminescence was proportional to ATP concentration when the luciferin + luciferase level was kept constant. The amount of protein in each sample was determined by the Lowry method. The ATP concentration in the plasmodium was determined for each sample by dividing the amount of ATP observed by the protein content. The water and protein contents of plasmodia were $83 \%$ and $7.8 \%$, respectively. Experiments were performed four times for each concentration of chemical. Standard deviations ranged from 5 to $10 \%$. All experiments were performed at room temperature $\left(19\right.$ to $\left.20^{\circ} \mathrm{C}\right)$.

\section{RESULTS}

\section{Changes in ATP concentration resulting from chemical stimulation}

The ATP concentration in plasmodia immersed in water decreased and attained a constant level within $8 \mathrm{~min}$. This was probably caused by anaerobic conditions. In the subsequent analysis, the ATP concentration 15 min after immersion in water was taken as standard; this ranged from 1.8 to $2.3 \mathrm{mM}$. The effect of immersion in various solutions on ATP concentrations relative to those in water is shown in Fig. 2. There was an increase in ATP concentration soon after immersion in $\mathrm{CaCl}_{2}$ and $\mathrm{KCl}$, but little change in glucose, $\mathrm{KH}_{2} \mathrm{PO}_{4}$ and sucrose. A slow decrease in ATP concentration occurred in ethanol or heavy water, and this was often accompanied by a release of yellow pigments into the medium. Hence, ethanol and heavy water may have toxic effects.

We have shown that contraction or relaxation in the plasmodium takes place about 15 min after chemical stimulation (Ueda et al., 1976) and we will therefore concentrate on the ATP concentration at this time.

\section{Chemically induced changes in ATP concentration in relation to contraction and chemotaxis}

Table 1 summarizes the effects of various chemicals on the ATP concentration $15 \mathrm{~min}$ after stimulation, along with references to their effects on chemotaxis and contraction. Repellent electrolytes induced an increase in ATP $\left(\mathrm{CaCl}_{2}, \mathrm{MgCl}_{2}, \mathrm{NaCl}\right.$ and $\left.\mathrm{KCl}\right)$. Hydrophobic repellents, such as ethanol, butyric acid, picrate and quinine, did not result in any marked change in ATP content. High concentrations of sucrose induced a large transient contraction and repulsion presumably due to their osmotic effect, but did not affect the ATP content. 


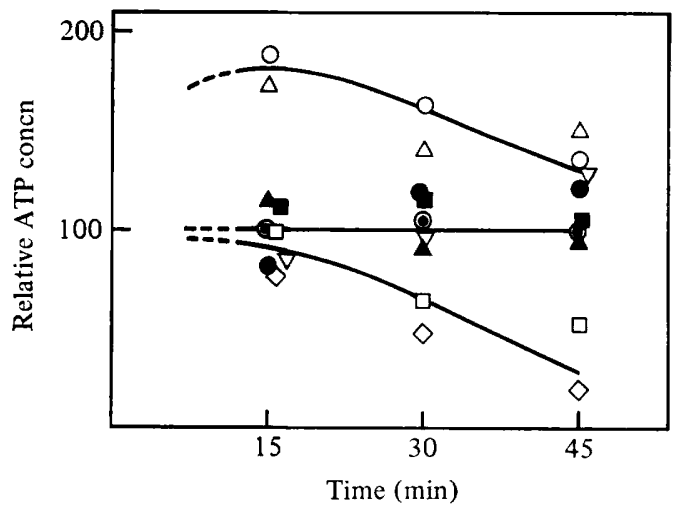

Fig. 2. Time course of the change in ATP concentration after chemical stimulation, relative to that in control plasmodia immersed in water for $15 \mathrm{~min}(100) . \mathrm{O}, \mathrm{CaCl}_{2}(3 \mathrm{mM}) ; \triangle, \mathrm{KCl}(20 \mathrm{mM})$; $\odot, \mathrm{H}_{2} \mathrm{O} ; 0$, glucose $(3 \mathrm{mM}) ; \boldsymbol{\Lambda}$, alanine $(3 \mathrm{mM}) ; \boldsymbol{\square}, \mathrm{KH}_{2} \mathrm{PO}_{4}(10 \mathrm{~mm}) ; \nabla$, sucrose $(300 \mathrm{~mm}) ;$ $\square$, ethanol $(400 \mathrm{~mm}) ; \diamond, \mathrm{D}_{2} \mathrm{O}(50 \%, \mathrm{v} / \mathrm{v})$.

Table 1. ATP concentrations in plasmodia after 15 min treatment with various chemicals

Repellents

Class

Chemical

Concn

Relative

(protoplasmic contraction)

(a) Electrolytes

$\mathrm{KCl}$
$\mathrm{NaCl}$
$\mathrm{CaCl}_{2}$
$\mathrm{MgCl}_{2}$
Ethanol
Acetaldehyde
Picrate
Butyric acid
Quinine
Sucrose
$\mathrm{D}_{2} \mathrm{O}$

(c) High osmotic pressure

(d) Heavy water

$\mathrm{D}_{2} \mathrm{O}$

Attractants

(protoplasmic relaxation)

$\begin{array}{lr}\text { Glucose } & 3 \\ \text { 2-Deoxyglucose } & 3 \\ \text { Alanine } & 3 \\ \text { Maltose } & 10 \\ \text { Phenylalanine } & 10 \\ \mathrm{KH}_{2} \mathrm{PO}_{4} & 10\end{array}$

20
20
3
3
400
10
3
1
1
300
$20 \%(\mathrm{v} / \mathrm{v})$
$50 \%(\mathrm{v} / \mathrm{v})$

$\begin{array}{rl}154 \pm 16 & 1,2 \\ 132 \pm 10 & \\ 151 \pm 10 & \\ 130 \pm 8 & \\ 96 \pm 12 & 3,4,5 \\ 100 \pm 3 & \\ 108 \pm 9 & \\ 113 \pm 5 & \\ 74 \pm 3 & \\ 85 \pm 5 & 2 \\ 97 \pm 4 & 6 \\ 72 \pm 10 & \end{array}$

$97 \pm 8$

$90 \pm 5$

$113 \pm 16$

$109 \pm 4$

$86 \pm 10$

$111 \pm 6$

$$
1,2,7,8,9
$$

* Relative to the value in water at $15 \mathrm{~min}$ which is taken as 100 .

$\dagger$ References to the effects of these chemicals on protoplasmic contraction or relaxation: 1, Ueda et al. (1975); 2, Ueda et al. (1976); 3, Ueda \& Kobatake (1977a); 4, Ueda \& Kobatake (1977b); 5, Ataka et al. (1978); 6, Götz von Olenhusen \& Wohlfarth-Bottermann (1979); 7, Knowles \& Carlile (1978); 8, Kincaid et al. (1978); 9, Chet et al. (1977).

There are thus two types of repellent, both of which act through causing contraction, but which differ in their effect on ATP concentration. This implies that there is more than one pathway by which the information sensed at the surface membrane is transmitted to cause the contraction.

The attractants examined (glucose, galactose, alanine, 2-deoxyglucose and $\mathrm{KH}_{2} \mathrm{PO}_{4}$ ) did not greatly affect the ATP concentration, whether they were electrolytes or non-electrolytes. 


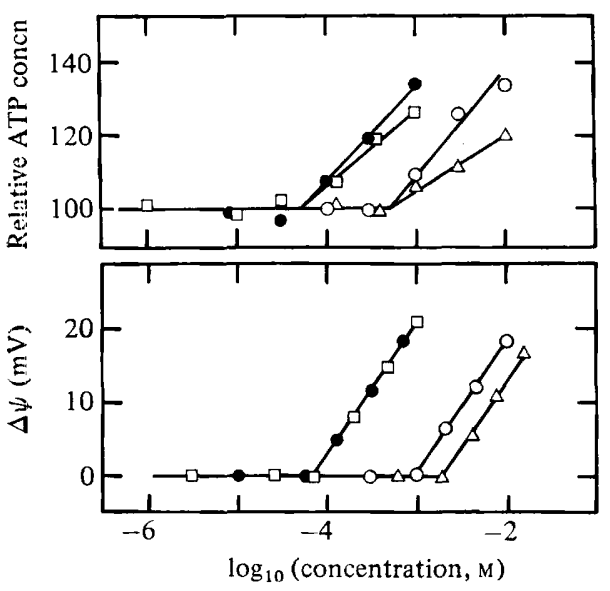

Fig. 3

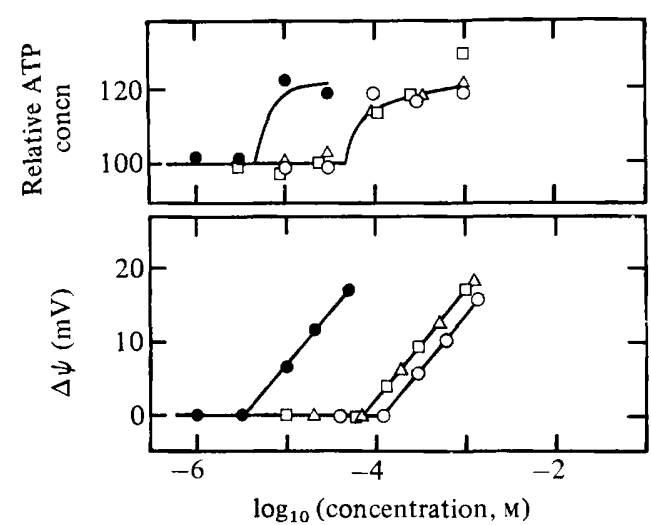

Fig. 4

Fig. 3. Dependence of the increase in ATP concentration and the membrane potential $(\Delta \psi)$ for the plasmodium of the American strain on the concentration of stimulant salt: $O, \mathrm{MgCl}_{2} ; \square$, $\mathrm{CaCl}_{2} ; \mathrm{O}, \mathrm{KCl} ; \triangle, \mathrm{NaCl}$.

Fig. 4. Dependence of the increase in ATP concentration and the membrane potential $(\Delta \psi)$ for the plasmodium of the albino strain on the concentration of stimulant salt: $\mathbf{O}, \mathrm{MgCl}_{2} ; \square, \mathrm{CaCl}_{2}$; $\mathrm{O}, \mathrm{KCl} ; \triangle, \mathrm{NaCl}$.

\section{ATP increase as a function of the concentration of stimulant salt}

Figure 3 shows the dependence of the ATP increase on the stimulant salt concentration for the plasmodium of the American strain. For comparison, corresponding data for the membrane potential (Ueda et al., 1975) are reproduced in the lower trace. The ATP concentration stayed constant until the concentration of each salt reached its respective threshold $\left(C_{\mathrm{th}}\right)$. Above $C_{\mathrm{th}}$, the intracellular ATP increased gradually with increase in salt concentration. The ATP increase reached 20 to $40 \%$ above the control at a concentration 10 times the respective $C_{\mathrm{th}}$. These changes paralleled those in membrane potential response as shown in the lower trace.

Figure 4 shows similar plots for the albino strain (LU647 $\times$ LU861), which had a threshold for membrane potential (Ueda \& Kobatake, 1979) and ATP increase at about one-tenth of the concentration effective for the American strain.

These results indicate that the ATP increase described above originates from the chemoreceptive process. Metabolizable chemicals which support the growth of the plasmodia, such as glucose, did not appreciably affect the ATP content.

\section{DISCUSSION}

Stimulation of the receptive membrane should alter a postulated mediator concentration, and changes in mediator concentration should modify the contractile activity. ATP has been shown, by an injection method, to cause contraction (Ueda \& Götz von Olenhusen, 1978; Ueda et al., 1978). Our present results show that stimulation causes changes in ATP concentrations. We therefore suggest that ATP is a mediator in the response to repellent salts.

Recently, Yoshimoto et al. (1980) found that the amount of ATP varied with rhythmic contraction and that ATP concentrations increased during the contraction phase. Since they monitored the ATP concentration indirectly, by measuring the ATP which leaked from the cell interior with chemical modification of the surface membrane, the variation in ATP in their experiment can be attributed to changes in either the permeability of the membrane or the intracellular concentration of ATP. If the latter is the explanation for their results, then their data and ours indicate that contraction and ATP increase coincide. 
Plasmodia contrast with skeletal muscle where the intracellular ATP concentration is kept constant by the regulating action of phosphocreatine systems (Curtin \& Davies, 1973). However, the lack of ATP-buffering system in the plasmodium is also found in other cells. For example, in nerve electroplaque synapses of Torpedo, ATP concentration paralleled the change in acetylcholine concentration (Israel et al., 1977). Thus, there is a possibility that ATP itself may work as a key substance in regulation.

We found the ATP concentration to be about $2 \mathrm{~mm}$ in the frontal region of the plasmodium, but a value of about $1 \mathrm{~mm}$ was reported by Hatano \& Takeuchi (1960). These values are much higher than that of $0.2 \mathrm{~mm}$ which was shown by injection studies to bring about the optimal contraction in the plasmodial strand (Ueda \& Götz von Olenhusen, 1978). This discrepancy might be due to differences between the plasmodial front and strands removed from the rear of a plasmodium. Sakai \& Kamiya (1976) found that the ATP concentration was highest in the frontal region and decreased gradually towards the rear.

\section{REFERENCES}

Ataka, M., Tsuchir, A., Ueda, T., Kurihara, K. \& Kobatake, Y. (1978). Comparative studies on the reception of bitter stimuli in the frog, Tetrahymena, slime mold and Nitella. Comparative Biochemistry and Physiology 61A, 109-115.

Beutler, E. \& Baluda, M. (1964). Simplified determination of blood adenosine triphosphate using the firefly system. Blood 23, 688-698.

CAMP, W. G. (1936). A method of cultivating myxomycete plasmodia. Bulletin of the Torrey Botanical Club 63, 205-210.

Carlile, M. J. (1970). Nutrition and chemotaxis in the myxomycete Physarum polycephalum: the effect of carbohydrates on the plasmodium. Journal of General Microbiology 63, 221-226.

Chet, I., Naveh, A. \& Henis, Y. (1977). Chemotaxis of Physarum polycephalum towards carbohydrates, amino acids and nucleotides. Journal of General Microbiology 102, 145-148.

Curtin, N. A. \& Davies, R. E. (1973). Chemical and mechanical changes during stretching of activated frog skeletal muscle. Cold Spring Harbor Symposia on Quantitative Biology 37, 619-626.

GÖTZ VON OLENHUSEN, K. \& WOHLFARTH-BOTTERMANN, K. E. (1979). Effects of caffeine and $\mathrm{D}_{2} \mathrm{O}$ on persistence and de novo generation of intrinsic oscillatory contraction automaticity in Physarum. Cell and Tissue Research 197, 479-499.

Hatano, S. \& TakeUChI, I. (1960). ATP content in myxomycete plasmodium and its levels in relation to some external conditions. Protoplasma 52, 169-183.

Hato, M., Ueda, T., Kurihara, K. \& Kobatake, Y. $(1976 a)$. Changes in zeta potential and membrane potential of slime mold Physarum polycephalum in response to chemical stimuli. Biochimica et biophysica acta 426, 73-80.

Hato, M., Ueda, T., Kurihara, K. \& Kobatake, Y. $(1976 b)$. Phototaxis in true slime mold Physarum polycephalum. Cell Structure and Function 1, 155-164.

ISHIDA, N., Kurihara, K. \& Kobatake, Y. (1977). Changes in adhesive properties of slime mold Physarum polycephalum accompanied with chemoreception. Cytobiologie 15, 269-274.
IShidA, N., KuRiHARA, K. \& Kobatake, Y. (1978). Dynamic conformational changes of surface membrane of true slime mold in response to chemoreception revealed by reactivity with chemical agents. Comparative Biochemistry and Physiology 60, 241-246.

Israel, M., Lesbats, B., Manaranche, R., Marsal, J., Mastour-Frachon, P. \& Meunier, F. M. (1977). Related changes in amount of ACh and ATP in resting and active Torpedo nerve electroplaque synapses. Journal of Neurochemistry 28, 1259-1267.

Kincaid, R. L. \& Mansour, T. E. (1978). Chemotaxis toward carbohydrates and aminoacids in Physarum polycephalum. Experimental Cell Research 116, 377-385.

KIncaId, R. L. \& Mansour, T. E. (1979). Cyclic 3',5'-AMP phosphodiesterase in Physarum polycephalum. I. Chemotaxis toward inhibitors and cyclic nucleotides. Biochimica et biophysica acta 588, 332-341.

Knowles, D. J. C. \& Carlile, M. J. (1978). The chemotactic response of plasmodia of the myxomycete Physarum polycephalum to sugars and related compounds. Journal of General Microbiology 108, 17-25.

Mito, Y., Kurihara, K. \& Kobatake, Y. (1980). Selective suppression of positive chemotaxis in Physarum polycephalum by treatment with rotenone or under anaerobic condition. European Journal of Cell Biology 21, 43-47.

SAKaI, T. \& KamiYA, N. (1976). ATP level in the plasmodium of Physarum polycephalum. 29th Annual Meeting of the Japan Society for Cell Biology Sendai, Japan, Abstracts, p. 46.

UEDA, T. \& GöTZ vON Olenhusen, K. (1978). Replacement of endoplasm with artificial media in plasmodial strands of Physarum polycephalum: effects on contractility and morphology. Experimental Cell Research 116, 55-62.

UEDA, T. \& KoBATAKE, Y. (1977a). Hydrophobicity of biosurfaces as shown by chemoreceptive thresholds in Tetrahymena, Physarum and Nitella. Journal of Membrane Biology 34, 351-368.

Ueda, T. \& Kobatake, Y. $(1977 b)$. Changes in 
membrane potential, zeta potential and chemotaxis of Physarum polycephalum in response to $\mathrm{n}$ alcohols, n-aldehydes and n-fatty acids. Cytobiologie 16, 16-26.

UEDA, T. \& KoBATAKe, Y. (1979). Spectral analysis of fluorescence of 8-anilino-1-naphthalenesulfonate in chemoreception with a white plasmodium of Physarum polycephalum: evidences for conformational change in chemoreceptive membrane. Biochimica et biophysica acta 557, 199-207.

Ueda, T., Terayama, K., Kurihara, K. \& KoBATAKE, Y. (1975). Threshold phenomena in chemoreception and taxis in slime mold Physarum polycephalum. Journal of General Physiology 65, 223-234.
Ueda, T., Muratsugu, M., Kurihara, K. \& Kobatake, Y. (1976). Chemotaxis in Physarum polycephalum: effects of chemicals on isometric tension of the plasmodial strand in relation to chemotactic movement. Experimental Cell Research 100, 337-344.

UEDA, T., GöTZ Von OlenHusen, K. \& WohlfaRTHBOTTERMANN, K. E. (1978). Reaction of the contractile apparatus in Physarum to injected $\mathrm{Ca}^{2+}$, ATP, ADP and 5' AMP. Cytobiologie 18, 76-94. Yoshimoto, Y., Matsumura, F., SAKaI, T. \& KAMIYA, N. (1980). Oscillations in $\mathrm{Ca}^{2+}$ and ATP effluxes coupled with tension generation in the plasmodial strand of Physarum. Cell Motility (in the Press). 